\title{
THE STRONG $c$-SYMMETRIC DISTRIBUTION
}

\author{
A. SRI RANGA \\ (Received 31 August 1990; revised 29 April 1991) \\ Communicated by P. C. Fenton
}

\begin{abstract}
In this paper, we consider a class of strong symmetric distributions, which we refer to as the strong $c$-symmetric distributions. We provide, as the main result of this paper, conditions satisfied by the recurrence relations of certain polynomials associated with these distributions.
\end{abstract}

1991 Mathematics subject classification (Amer. Math. Soc.): 30 E 05, 33 A 65.

\section{Introduction}

A strong distribution, $d \psi(t)$, is one for which the moments

$$
\mu_{n}=\int_{\mathbf{R}} t^{n} d \psi(t),
$$

exist and are finite for $n=0, \pm 1, \pm 2, \ldots$. We believe that the first to consider such distributions were Jones et al. [1], in relation to the moment problem which they referred to as the "strong moment problem." Recently, in Sri Ranga [3] and in Sri Ranga and McCabe [4], extensive studies have been made on certain polynomials associated with such distributions, and in particular, on the recurrence relations satisfied by these polynomials. These polynomials are very much related to the Laurent polynomials considered for example in [2].

Here, first we present some results, a good part of which are given in [4], and the rest easily follow from these.

This research was supported by CNPq of Brazil.

(C) 1992 Australian Mathematical Society 0263-6115/92 \$A2.00+0.00 
For any strong distribution, $d \psi(t)$, the relations

(i) $Q_{n}(z)$ is a monic polynomial of degree $n$, for $n \geq 0$,

(ii) $\int_{\mathbf{R}} t^{-2[n / 2]+s} Q_{n}(t) d \psi(t)=0, \quad 0 \leq s \leq n-1$, for $n \geq 1$,

uniquely define the (monic) polynomials $Q_{n}(z)$, for $n \geq 0$. Here, [x] represents the integral part of $x$.

If the Hankel determinants $H_{s}^{(k)}=\operatorname{det}\left(\mu_{k+j+m}\right)_{j, m=0}^{s-1}$, associated with the moments of this distribution satisfy

$$
H_{2 n}^{(-2 n+1)} \neq 0, \quad n \geq 0,
$$

then the polynomials $Q_{n}(z)$ satisfy the recurrence relations

$$
\begin{aligned}
& Q_{2 n}(z)=\left(z-\beta_{2 n}\right) Q_{2 n-1}(z)-\alpha_{2 n} Q_{2 n-2}(z), \\
& Q_{2 n+1}(z)=\left\{\left(1+\alpha_{2 n+1}\right) z-\beta_{2 n+1}\right\} Q_{2 n}(z)-\alpha_{2 n+1} z^{2} Q_{2 n-1}(z),
\end{aligned}
$$

for $n \geq 1$, where $Q_{0}(z)=1, Q_{1}(z)=\left(z-\beta_{1}\right), \alpha_{n+1}>0$ and $\beta_{n} \in \mathbb{R}$.

If this distribution is symmetric (about the origin), i.e., $d \psi(t)=-d \psi(-t)$, then its Hankel determinants satisfy

$$
H_{2 r+1}^{(2 m+1)}=0 \text { and }(-1)^{r} H_{2 r}^{(2 m+1)}>0,
$$

for $r \geq 0$ and $m=0, \pm 1, \pm 2, \ldots$. As a result, the polynomials $Q_{n}(z)$ satisfy the recurrence relations (1.2), and in these recurrence relations, the coefficients $\beta_{n}$ are zero for all $n \geq 1$. Hence, for example, we have for any strong symmetric distribution

$$
Q_{2 n}(0) \neq 0, \quad n \geq 0,
$$

and

$$
\int_{\mathbf{R}} t^{-2 n-1} Q_{2 n}(t) d \psi(t)=0, \quad n \geq 0 .
$$

In the three examples of strong symmetric distributions given in [4], namely, the strong Tchebycheff, the strong Legendre and the strong Hermite distributions, we note that the coefficients $\alpha_{n}$ and $\beta_{n}$ of the associated recurrence relations satisfy, in addition to the expected condition $\beta_{n}=0, n \geq 1$, the condition that $\alpha_{2 n}$ are constant for all $n \geq 1$. We show here that this is due to these distributions being members of a "special" class of strong symmetric distributions.

\section{The strong $c$-symmetric distributions}

We call any strong symmetric distribution, $d \psi(t)$, for which there corresponds a constant $c$ such that

$$
d \psi(t) / t=-d \psi(c / t) /(c / t)
$$


for all $t \in \mathbb{R}$, a "strong $c$-symmetric distribution."

For a strong $c$-symmetric distribution $d \psi(t)$, the following two conditions hold.

(a) If one can write the increment of $\psi(t)$ at the point $t$ as $\rho(t)$, then $\rho(t) / t=\rho(c / t) /(c / t)$. This relation characterizes the strong $c$-symmetric distribution in all the points where $\psi(t)$ has step increments.

(b) If $d \psi(t)$ can be given as $w(t) d t$ in the neighbourhood of some point $\tilde{t}$ (possible wherever $\psi(t)$ is continuous and differentiable), then the same holds in the neighbourhood of $c / \tilde{t}$, and the function $w(t)$ satisfies $w(t)=$ $w(c / t)$.

It is easily verified that the examples of strong symmetric distributions given in [4] are strong $c$-symmetric distributions (with $c=a b$ in the first two examples and $c=a$ in the other).

For any strong $c$-symmetric distribution $d \psi(t)$, we have from (2.1), with the substitution $t=c / u$, that

$$
\int_{0}^{\sqrt{c}} t^{m} d \psi(t)=c^{m+1} \int_{\sqrt{c}}^{\infty} u^{-m-2} d \psi(u), \quad m=0, \pm 1, \pm 2, \ldots
$$

This result together with the symmetry of $d \psi(t)$ yields for the moments

$$
\mu_{m}=c^{m+1} \mu_{-m-2}, \quad m \geq 0 .
$$

For any polynomial $Q(t)=q_{0}+q_{1} t+\cdots+q_{l} t^{l}$, we can write

$$
\int_{\mathbf{R}} t^{m} Q(t) d \psi(t)=\sum_{r=0}^{l} q_{r} \mu_{m+r}, \quad m=0, \pm 1, \pm 2, \ldots
$$

Hence, the relation (2.2) gives immediately that

$$
\int_{\mathbf{R}} t^{m} Q(t) d \psi(t)=c^{m+1} \int_{\mathbf{R}} t^{-m-2} Q(c / t) d \psi(t), \quad m=0, \pm 1, \pm 2, \ldots
$$

We now give the principal result of this article.

THEOREM 3.1. For any strong c-symmetric distribution $d \psi(t)$, the polynomials $Q_{n}(z), n \geq 0$, defined by (1.1), satisfy the recurrence relation (1.2), with

$$
\beta_{n}=0, \quad \alpha_{2 n}=c, \quad \text { for } n \geq 1 .
$$

Proof. As we have already mentioned, the result that $Q_{n}(z)$ satisfy the recurrence relations (1.2) and that $\beta_{n}=0$, for $n \geq 1$, follows from the symmetry of $d \psi(t)$. To prove the other, we have from (2.3) that

$$
\int_{\mathbf{R}} t^{-2 n+s} Q_{2 n}(t) d \psi(t)=c^{-2 n+s+1} \int_{\mathbf{R}} t^{2 n-s-2} Q_{2 n}(c / t) d \psi(t),
$$


for $s=0, \pm 1, \pm 2, \ldots$. Hence, from (1.1) and (1.4), one can obtain

$$
\int_{\mathbf{R}} t^{-s-2}\left\{t^{2 n} Q_{2 n}(c / t)\right\} d \psi(t)=0, \quad-1 \leq s \leq 2 n-2 .
$$

This relation is also true for $s=2 n-1$. However, as we do not need this for the rest of our analysis, we have omitted it in the proof.

From (1.3), since $Q_{2 n}(0) \neq 0$, dividing the above relation by $Q_{2 n}(0)$ and then replacing $s$ by $2 n-2-s$, we obtain

$$
\int_{\mathbf{R}} t^{-2 n+s}\left\{t^{2 n} Q_{2 n}(c / t) / Q_{2 n}(0)\right\} d \psi(t)=0, \quad 0 \leq s \leq 2 n-1 .
$$

This relation is equivalent to (1.1), with even values of $n$ only, and where, in place of the monic polynomials $Q_{2 n}(z)$, one finds the monic polynomials $z^{2 n} Q_{2 n}(c / z) / Q_{2 n}(0)$. Hence, as (1.1) uniquely defines its polynomials, we have

$$
Q_{2 n}(z)=z^{2 n} Q_{2 n}(c / z) / Q_{2 n}(0), \quad n \geq 0 .
$$

An immediate consequence of this result is that if $\tilde{z}$ is a zero of $Q_{2 n}(z)$ then so are $c / \tilde{z},-\tilde{z}$ and $-c / \tilde{z}$. Here, we have also considered the evenness of $Q_{2 n}(z)$.

With $\beta_{n}=0, n \geq 1$, we obtain from the recurrence relation (1.2)

$$
Q_{2 n}(z)=\left(z^{2}-\alpha_{2 n}\right) Q_{2 n-2}(z)-\alpha_{2 n-2} \alpha_{2 n-1} z^{2} Q_{2 n-4}(z),
$$

for $n \geq 2$, with $Q_{0}(z)=1, Q_{2}(z)=z^{2}-\alpha_{2}$.

In (2.5), letting $z=0$ gives

$$
Q_{2 n}(0)=-\alpha_{2 n} Q_{2 n-2}(0)=(-1)^{n} \prod_{r=1}^{n} \alpha_{2 r}, \quad n \geq 1 .
$$

Again in (2.5), replacing $z$ by $c / z$, and then multiplying the resulting equation by $z^{2 n} / Q_{2 n}(0)$, one obtains

$$
\begin{aligned}
\left\{\frac{z^{2 n} Q_{2 n}(c / z)}{Q_{2 n}(0)}\right\}= & \left(z^{2}-\frac{c^{2}}{\alpha_{2 n}}\right)\left\{\frac{z^{2 n-2} Q_{2 n-2}(c / z)}{Q_{2 n-2}(0)}\right\} \\
& -\frac{\alpha_{2 n-1}}{\alpha_{2 n}} c^{2} z^{2}\left\{\frac{z^{2 n-4} Q_{2 n-4}(c / z)}{Q_{2 n-4}(0)}\right\},
\end{aligned}
$$

for $n \geq 1$, with $z^{0} Q_{0}(c / z) / Q_{0}(0)=1, z^{2} Q_{2}(c / z) / Q_{2}(0)=z-c^{2} / \alpha_{2}$.

Therefore, with result (2.4), we obtain from this recurrence relation

$$
Q_{2 n}(0)=-\frac{c^{2}}{\alpha_{2 n}} Q_{2 n-2}(0), \quad n \geq 1 \text {. }
$$


Comparing this with (2.6) we obtain $\alpha_{2 n}=c$, for $n \geq 1$, and this completes the proof of the theorem.

\section{References}

[1] W. B. Jones, W. J. Thron and H. Waadeland, 'A strong Stieltjes moment problem', Trans. Amer. Math. Soc. 261 (1980), 503-528.

[2] Olav Njastad and W. J. Thron, 'Unique solvability of the strong Hamburger moment problem', J. Austral. Math. Soc. (Series A) 40 (1986), 5-19.

[3] A. Sri Ranga, 'On a recurrence formula associated with strong distributions', SIAM $J$. Math. Anal. 21 (1990), 1335-1348.

[4] A. Sri Ranga and J. H. McCabe, 'On the extensions of some classical distributions', Proc. Edinburgh Math. Soc.. 34 (1991), 19-29.

Departamento de Ciências de Computação e Estatística

IBILCE, UNESP-Universidade Estadual Paulista

São José do Rio Preto, SP

Brazil 